\title{
A SIMPLE SUBSPACE IDENTIFICATION METHOD OF CLOSED-LOOP SYSTEMS USING ORTHOGONAL DECOMPOSITION
}

\author{
Tohru Katayama * Hideyuki Tanaka* Takeya Enomoto* \\ * Department of Applied Mathematics and Physics, \\ Graduate School of Informatics, Kyoto University, \\ Kyoto, 606-8501, Japan. \\ \{katayama,htanaka\}@amp.i.kyoto-u.ac.jp
}

\begin{abstract}
A simple method of identifying plant and controller operating in closed-loop is developed by the orthogonal decomposition technique. Under the assumption that the system is open-loop as well as closed-loop stable, a subspace identification algorithm of identifying the deterministic part is derived based on two successive LQ decompositions. The idea is somewhat related to the two-stage method (Van den Hof and Schrama, 1993) and to the projection method (Forssell and Ljung, 2000); but the present subspace based method can easily be applied to multivariable systems. Also, the algorithm is quite simple and has high accuracy. Some simulation results show that the present method yields better numerical results compared with a method based on joint input-output approach, especially if the disturbance is a colored noise. Copyright ${ }^{\odot} 2005$ IFAC
\end{abstract}

Keywords: Closed-loop systems, Subspace identification method, Orthogonal decomposition (ORT) method, LQ decomposition.

\section{INTRODUCTION}

The identification problem for closed-loop systems has received much interest recently (Chiuso and Picci, 2003; Codrons et al., 2002; Forssell and Ljung, 1999; Qin and Ljung, 2003; Jansson, 2003; Van den Hof and Schrama, 1993; Verhaegen, 1993), since there are many cases where open-loop experiments are impossible because of the problem of safety, stability, etc. (Van den Hof, 1997; Forssell and Ljung, 1999). A main difficulty in the identification of the closed loop system is due to the existence of correlation between plant inputs and disturbances, resulting in biased estimates of the plant parameters.

Closed loop identification methods are divided into three groups: (i) direct approach, (ii) indirect approach, and (iii) joint input-output approach. These approaches have advantages and disadvantages, see (Forssell and Ljung, 1999).
In fact, the direct approach is simple and useful because it ignores the feedback loop, although the estimate is biased due to a possible correlation between the disturbance and the input. For the indirect approach, the knowledge of the controller transfer function is needed. Moreover, since the estimates of the plant are of higher order, we need some model reduction procedures. The joint input-output approach has merit in that no knowledge of the controller is needed and an estimate of the plant is unbiased; however, the dimension of the estimated plant becomes higher as in the indirect approach.

In this paper, under the assumption that the plant and controller are open-loop stable, we present a simple closed-loop identification procedure based on the orthogonal decomposition (ORT) method developed in (Picci and Katayama, 1996b; Picci and Katayama, 1996a). This method has at least two advantages: one is that it can be naturally applied to MIMO systems, 
and the other is that the present method does not include a model reduction step needed in the joint inputoutput approach (Katayama et al., 2002; Katayama et al., 2005).

This paper is organized as follows. Section 2 presents the problem formulation, and in Section 3, the joint input-output data is decomposed into the deterministic and stochastic components. In Section 4, we derive a subspace identification method for closed-loop systems based on the deterministic component. Some numerical results are included in Section 5. Section 6 concludes the paper.

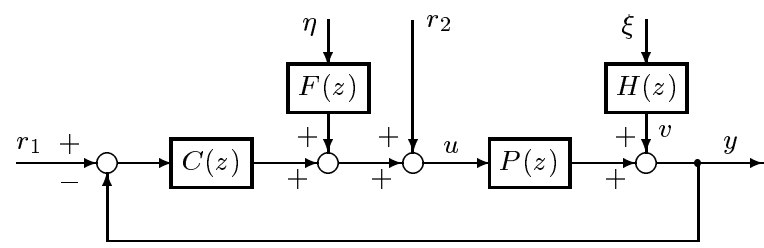

Fig. 1. Closed-loop system.

\section{PROBLEM FORMULATION}

Consider the identification of the closed-loop system shown in Fig. 1. It is assumed that the plant is timeinvariant and is given by

$$
y(t)=P(z) u(t)+H(z) \xi(t)
$$

where $P(z)$ and $H(z)$ are the plant and the noise filter, respectively. The input and output of the plant $P(z)$ are $u \in \mathbb{R}^{m}$ and $y \in \mathbb{R}^{p}$, respectively, $\xi \in \mathbb{R}^{p}$ is a white noise with mean 0 and covariance matrix $\Lambda_{\xi}>0$, and $H(z)$ is a minimum phase transfer function satisfying $H(\infty)=I_{p}$. The control signal is generated by

$$
u(t)=r_{2}(t)+C(z)\left[r_{1}(t)-y(t)\right]+F(z) \eta(t)
$$

where external inputs $r_{1} \in \mathbb{R}^{m}$ and $r_{2} \in \mathbb{R}^{p}$ are set points or test signals for identification, and where $\eta \in \mathbb{R}^{p}$ is a white noise with mean 0 and covariance matrix $\Lambda_{\eta}>0$, and where $F(z)$ is a minimum phase transfer function satisfying $F(\infty)=I_{m}$.

We make assumptions for the feedback system.

A1: We assume that the feedback system is wellposed in the sense that $(u, y)$ are determined uniquely. This condition is certainly satisfied if $I_{p}+P(\infty) C(\infty)$ and $I_{m}+C(\infty) P(\infty)$ are nonsingular. For simplicity, we assume that the plant is strictly proper, i.e. $P(\infty)=0$.

A2: The feedback system is stable, and the plant and controller are also stable.

A3: The external inputs $\left(r_{1}, r_{2}\right)$ satisfy PE conditions and are uncorrelated with white noises $\xi$ and $\eta$.

A4: There is no feedback from $(u, y)$ to $\left(r_{1}, r_{2}\right)$.
A5: External inputs and noises $\left(r_{1}, r_{2}, \xi, \eta\right)$ are 2 ndorder jointly stationary processes with mean zero.

The present identification problem of closed-loop system is stated as follows.

Identification of Closed-Loop Systems: We derive a subspace method to identify the state space model of the plant $P(z)$ and controller $C(z)$ based on a finite measurement data $\left\{r_{1}(t), r_{2}(t), u(t), y(t), t=\right.$ $1,2, \cdots, N+2 k-1\}$, where $k>n$.

\section{ORTHOGONAL DECOMPOSITION IN HILBERT SPACE: BASIC IDEA}

In this section, we compute the deterministic components of the joint input-output process $w=(u, y)$ in the closed-loop system based on the ORT method (Picci and Katayama, 1996b).

Define $r=\left[\begin{array}{l}r_{1} \\ r_{2}\end{array}\right]$ and $w=\left[\begin{array}{l}u \\ y\end{array}\right]$. Let $\mathcal{R}$ and $\mathcal{W}$ denote vector spaces obtained by taking all finite linear combinations of $r$ and $w$, respectively. Also we define $\mathcal{H}:=\mathcal{R} \vee \mathcal{W}$ by all finite linear combinations of $r$ and $w$. Then we obtain a Hilbert space by closing the vector space $\mathcal{H}$ with respect to the norm induced by the scalar product $\langle\alpha, \beta\rangle=E\{\alpha \beta\}$ for $\alpha, \beta \in \mathcal{H}$, where $E\{\cdot\}$ denotes the mathematical expectation. The orthogonal projection onto the subspace $\mathcal{A}$ is denoted by the symbol $\hat{E}\{\cdot \mid \mathcal{A}\}$.

Let $t$ be the present time. We define the linear spaces of 2 nd-order random variables spanned by the infinite past and future of the exogenous input vector at $t$ as

$$
\mathcal{R}_{t}^{-}:=\overline{\operatorname{span}}\{r(\sigma) \mid \sigma<t\}
$$

and

$$
\mathcal{R}_{t}^{+}:=\overline{\operatorname{span}}\{r(\sigma) \mid \sigma \geq t\}
$$

Then, we see that $\mathcal{R}=\mathcal{R}_{t}^{-} \vee \mathcal{R}_{t}^{+}$. Moreover, we define $\mathcal{W}_{t}^{-}$and $\mathcal{W}_{t}^{+}$similarly.

From Assumption A4, there is no feedback from the output $w$ to the input $r$. The feedback-free condition is that the future of $r$ is conditionally uncorrelated with the past of $w$ given the past of $r$, so that this condition can be written as

$$
\mathcal{R}_{t}^{+} \perp \mathcal{W}_{t}^{-} \mid \mathcal{R}_{t}^{-}
$$

Also from Assumption A3, the input $r$ satisfies the PE condition, which is equivalent to the fact that the input space $\mathcal{R}$ has a direct decomposition

$$
\mathcal{R}=\mathcal{R}_{t}^{-}+\mathcal{R}_{t}^{+}, \quad \mathcal{R}_{t}^{-} \cap \mathcal{R}_{t}^{+}=\{0\}
$$

This is guaranteed if the spectral density matrix $\Phi_{r}(z)$ of $r$ is positive definite on the unit circle $(|z|=1)$.

According to (Picci and Katayama, 1996b), it follows from the condition (3) that

$$
\hat{E}\left\{\mathcal{W}_{t}^{-} \mid \mathcal{R}\right\}=\hat{E}\left\{\mathcal{W}_{t}^{-} \mid \mathcal{R}_{t}^{-}\right\}, \quad t=0, \pm 1, \cdots
$$


In particular, since $w(t) \in \mathcal{W}_{t+1}^{-}$, we have

$$
\hat{E}\{w(t) \mid \mathcal{R}\}=\hat{E}\left\{w(t) \mid \mathcal{R}_{t+1}^{-}\right\}
$$

implying that the smoothed estimate $\hat{E}\{w(t) \mid \mathcal{R}\}$ is causal. Thus it can be expressed as a linear combination of the present and past inputs.

Now we define

$$
w_{d}(t)=\hat{E}\{w(t) \mid \mathcal{R}\}=\hat{E}\left\{w(t) \mid \mathcal{R}_{t+1}^{-}\right\}
$$

Then we see that $w_{d}(t)$ is a part of $w$ that is linearly related to the past inputs $r$; thus $w_{d}(t)$ is expressed as a moving average of $r(t), r(t-1), \cdots$. Hence, $w_{d}$ is called the deterministic component of $w$. The orthogonal projection of $w$ onto the complementary space $\mathcal{R}^{\perp}$, called the stochastic component of $\mathcal{W}$, is given by

$$
\begin{aligned}
w_{s}(t) & =w(t)-w_{d}(t) \\
& =w(t)-\hat{E}\{w(t) \mid \mathcal{R}\}
\end{aligned}
$$

This shows that the estimation error is uncorrelated with the whole history of the exogenous input $r$.

Lemma 1. (Picci and Katayama, 1996b) Under the assumption that the exogenous inputs are feedbackfree, the output process $w$ has the orthogonal decomposition

$$
w(t)=w_{d}(t)+w_{s}(t)
$$

where

$$
E\left\{w_{s}(t) w_{d}^{\mathrm{T}}(\tau)\right\}=0, \quad \forall t, \tau=0, \pm 1, \cdots
$$

Applying this decomposition results to the feedback system shown in Fig. 1, we have equations satisfied by the deterministic and stochastic components.

Lemma 2. The deterministic and stochastic components respectively satisfy the independent equations

$$
\begin{aligned}
& y_{d}(t)=P(z) u_{d}(t) \\
& u_{d}(t)=r_{2}(t)+C(z)\left[r_{1}(t)-y_{d}(t)\right]
\end{aligned}
$$

and

$$
\begin{aligned}
& y_{s}(t)=P(z) u_{s}(t)+H(z) \xi(t) \\
& u_{s}(t)=-C(z) y_{s}(t)+F(z) \eta(t)
\end{aligned}
$$

Proof. From (1), (2) and (7),

$$
\begin{aligned}
y_{d}(t)+y_{s}(t)= & P(z)\left[u_{d}(t)+u_{s}(t)\right]+H(z) \xi(t) \\
u_{d}(t)+u_{s}(t)= & r_{2}(t)+C(z)\left[r_{1}(t)-y_{d}(t)-y_{s}(t)\right] \\
& +F(z) \eta(t)
\end{aligned}
$$

Since $\xi, \eta, y_{s}, u_{s}$ are orthogonal to $\mathcal{R}$, the orthogonal projections of the above equations onto $\mathcal{R}$ and $\mathcal{R} \perp$ yield (8) and (9), respectively.

Remark 1. We see that the realization of deterministic and stochastic components are decoupled, since the two components are mutually uncorrelated. It should be, however, noted that though true for ideal case with infinite data, this observation is not true practically. For, in the case of finite input-output data, the estimate of the stochastic component $w_{s}$ is influenced by the unknown initial condition associated with the estimate of the deterministic component $w_{d}$ as discussed in (Picci and Katayama, 1996a); however the effect due to unknown initial conditions surely decreases for sufficiently long data.

Remark 2. Since $P(z)$ and $C(z)$ are stable, we can apply the ORT method to (8) to obtain the state space realizations of these transfer matrices. Now suppose that $P(z)$ and $C(z)$ are so identified. Then, from (9), we have

$$
\tilde{y}_{s}(t):=y_{s}(t)-P(z) u_{s}(t)=H(z) \xi(t)
$$

and

$$
\tilde{u}_{s}(t):=u_{s}(t)+C(z) y_{s}(t)=F(z) \eta(t)
$$

Applying the stochastic subspace identification method due to (Katayama and Picci, 1999), we can identify the noise models $H(z)$ and $F(z)$ from realizations of the stationary processes $\tilde{y}_{s}$ and $\tilde{u}_{s}$, respectively.

\section{IDENTIFICATION OF OPEN-LOOP SYSTEMS}

From (8), we have two deterministic equations

$$
y_{d}(t)=P(z) u_{d}(t)
$$

and

$$
u_{d}(t)-r_{2}(t)=C(z)\left[r_{1}(t)-y_{d}(t)\right]
$$

We define

$$
\tilde{y}_{d}(t)=y_{d}(t)-r_{1}(t), \quad \tilde{u}_{d}(t)=u_{d}(t)-r_{2}(t)
$$

Then, the plant and controller are expressed as the block diagrams shown in Fig. 2.

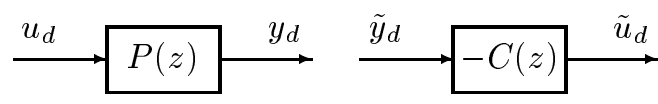

Fig. 2. Plant and controller in terms of deterministic components.

Notice that these are relations satisfied by deterministic components $\left(u_{d}, y_{d}\right)$ and $\left(\tilde{u}_{d}, \tilde{y}_{d}\right)$, where the noise components are removed in these relations. Thus Fig. 2 displays two independent open-loop systems for the plant and controller, so that we can use these relations to identify the open-loop plant $P(z)$ and the controller $C(z)$. This idea is closely related to the two-stage method (Van den Hof and Schrama, 1993) and the projection method (Forssell and Ljung, 1999), therein the sensitivity function of the closed-loop system is identified by using ARMA or FIR models to generate filtered estimate of the input process $u$.

Thus the scenario of closed-loop identification with an open-loop stable plant and/or controller is summarized as follows. 


\section{Identification algorithm of plant and controller}

Step 1: By using LQ decomposition, we compute the deterministic components of the joint input-output process $\left(y_{d}, u_{d}\right)$ and then compute $\left(\tilde{y}_{d}, \tilde{u}_{d}\right)$.

Step 2: Apply the ORT method to the input-output data $\left(u_{d}, y_{d}\right)$ to compute $P(z)=\left(A_{p}, B_{p}, C_{p}\right)$.

Step 3: Apply the ORT method to the input-output data $\left(\tilde{u}_{d}, \tilde{y}_{d}\right)$ to compute the controller transfer matrix $C(z)=\left(A_{c}, B_{c}, C_{c}, D_{c}\right)$.

\section{SUBSPACE IDENTIFICATION METHOD}

In this section, we derive a subspace identification method of the plant following the scenario described above. The subspace identification of the controller is similar.

Let the order of the plant be $n$. We see that any basis vector $x_{d}(t)$ yields a state space representation of $y_{d}(t)$, i.e.,

$$
\begin{aligned}
x_{d}(t+1) & =A x_{d}(t)+B u_{d}(t) \\
y_{d}(t) & =C x_{d}(t)+D u_{d}(t)
\end{aligned}
$$

where $A \in \mathbb{R}^{n \times n}$, and the estimate of the plant is given by $P(z)=C(z I-A)^{-1} B+D^{1}$.

Suppose that we are given the data $r(t), u(t), y(t)$ for $t=1,2, \cdots, N+k-1$. As usual, we define a block Hankel matrix

$$
\left[\frac{R_{p}}{R_{f}}\right]=\left[\begin{array}{cccc}
r(1) & r(2) & \cdots & r(N) \\
r(2) & r(3) & \cdots & r(N+1) \\
\vdots & \vdots & \ddots & \vdots \\
r(k) & r(k+1) & \cdots & r(k+N-1) \\
\hline r(k+1) & r(k+2) & \cdots & r(k+N) \\
r(k+2) & r(k+3) & \cdots & r(k+N+1) \\
\vdots & \vdots & \ddots & \vdots \\
r(2 k) & r(2 k+1) & \cdots & r(2 k+N-1)
\end{array}\right]
$$

and $U_{p}, U_{f}$ and $Y_{p}, Y_{f}$ are defined in the same way. Moreover, we define data matrices $R, U$ and $Y$ as

$$
\begin{aligned}
R & =\left[\begin{array}{l}
R_{p} \\
R_{f}
\end{array}\right] \in \mathbb{R}^{2 k(p+m) \times N} \\
U & =\left[\begin{array}{l}
U_{f} \\
U_{p}
\end{array}\right] \in \mathbb{R}^{2 k m \times N} \\
Y & =\left[\begin{array}{l}
Y_{p} \\
Y_{f}
\end{array}\right] \in \mathbb{R}^{2 k p \times N}
\end{aligned}
$$

From (12), we have a matrix input-output equation

$$
\hat{Y}_{f}=\mathcal{O}_{k} \hat{X}_{k}+\Psi_{k} \hat{U}_{f}
$$

where $\left({ }^{\wedge}\right)$ denotes the deterministic component, and $\mathcal{O}_{k}, \Psi_{k}$ and $\hat{X}_{k}$ are given by

\footnotetext{
1 Since $P(z)$ is assumed to be strictly proper, the actual $D$ matrix
} is zero.

$$
\begin{aligned}
& \mathcal{O}_{k}=\left[\begin{array}{c}
C \\
C A \\
\vdots \\
C A^{k-1}
\end{array}\right] \\
& \Psi_{k}=\left[\begin{array}{cccc}
D & 0 & \cdots & 0 \\
C B & D & \cdots & 0 \\
\vdots & \vdots & \ddots & \vdots \\
C A^{k-2} B & C A^{k-3} B & \cdots & D
\end{array}\right] \\
& \hat{X}_{k}=\left[x_{d}(k) x_{d}(k+1) \cdots x_{d}(k+N-1)\right]
\end{aligned}
$$

The algorithm based on the ORT method is described as follows.

\section{Identification Algorithm - ORT method}

Step 1: Compute LQ decomposition

$$
\left[\begin{array}{c}
R \\
U \\
Y
\end{array}\right]=\left[\begin{array}{ccc}
M_{11} & 0 & 0 \\
M_{21} & M_{22} & 0 \\
M_{31} & M_{32} & M_{33}
\end{array}\right]\left[\begin{array}{l}
Q_{1}^{T} \\
Q_{2}^{T} \\
Q_{3}^{T}
\end{array}\right]
$$

Then the deterministic components are given by

$$
\left[\begin{array}{c}
\hat{U} \\
\hat{Y}
\end{array}\right]=\left[\begin{array}{c}
\hat{U}_{f} \\
\hat{U}_{p} \\
\hat{Y}_{p} \\
\hat{Y}_{f}
\end{array}\right]=\left[\begin{array}{l}
M_{21} \\
M_{31}
\end{array}\right] Q_{1}^{T}
$$

Step 2: Compute the LQ decomposition

$$
\left[\begin{array}{c}
\hat{U}_{f} \\
\hat{U}_{p} \\
\hat{Y}_{p} \\
\hat{Y}_{f}
\end{array}\right]=\left[\begin{array}{cccc}
L_{11} & 0 & 0 & 0 \\
L_{21} & L_{22} & 0 & 0 \\
L_{31} & L_{32} & L_{33} & 0 \\
L_{41} & L_{42} & L_{43} & L_{44}
\end{array}\right]\left[\begin{array}{c}
\hat{Q}_{1}^{T} \\
\hat{Q}_{2}^{T} \\
\hat{Q}_{3}^{T} \\
\hat{Q}_{4}^{T}
\end{array}\right]
$$

Again, using the idea of the ORT, we obtain the "deterministic part" as

$$
\left[\begin{array}{c}
\hat{U}_{f} \\
\hat{U}_{p} \\
\hat{Y}_{p} \\
\hat{Y}_{f}
\end{array}\right]=\left[\begin{array}{cc}
L_{11} & 0 \\
L_{21} & L_{22} \\
L_{31} & L_{32} \\
L_{41} & L_{42}
\end{array}\right]\left[\begin{array}{l}
\hat{Q}_{1}^{T} \\
\hat{Q}_{2}^{T}
\end{array}\right]
$$

Step 3: Combining (13) and (16), $\hat{Y}_{f}$ is expressed as

$$
L_{41} \hat{Q}_{1}^{T}+L_{42} \hat{Q}_{2}^{T}=\mathcal{O}_{k} \hat{X}_{k}+\Psi_{k} L_{11} \hat{Q}_{1}^{T}
$$

Post-multiplying (17) by $\hat{Q}_{2}$ yields $L_{42}=\mathcal{O}_{k} \hat{X}_{k} \hat{Q}_{2}$. Under the assumption that $\hat{X}_{k} \hat{Q}_{2}$ has row full rank, we have

$$
\operatorname{Im}\left(\mathcal{O}_{k}\right)=\operatorname{Im}\left(L_{42}\right)
$$

Compute the SVD of $L_{42}$ as

$$
\begin{aligned}
L_{42} & =\left[\begin{array}{ll}
U_{1} & U_{2}
\end{array}\right]\left[\begin{array}{cc}
\Sigma_{1} & 0 \\
0 & \Sigma_{2}
\end{array}\right]\left[\begin{array}{l}
V_{1}^{T} \\
V_{2}^{T}
\end{array}\right] \\
& =U_{1} \Sigma_{1} V_{1}^{T}+U_{2} \Sigma_{2} V_{2}^{T} \approx U_{1} \Sigma_{1} V_{1}^{T}
\end{aligned}
$$

Then, we define the extended observability matrix as

$$
\mathcal{O}_{k}:=U_{1} \Sigma_{1}^{\frac{1}{2}}
$$




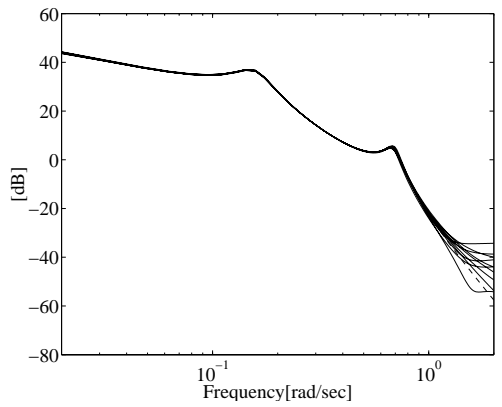

Fig. 3. Bode plots of the estimated plant by the present method (white noise).

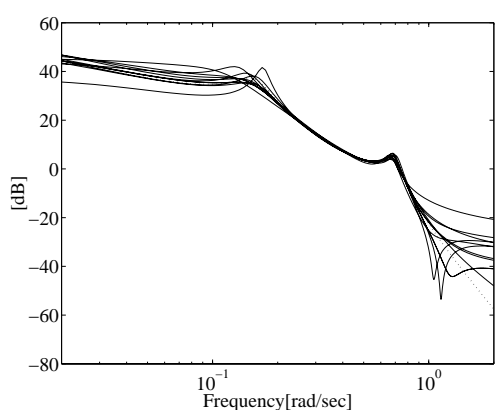

Fig. 4. Bode plots of the estimated plant by the joint input-output method (white noise).

Step 4: We see that $(C, A)$ are obtained by using the shift-invariance property of the observability matrix $\mathcal{O}_{k}$. Pre-multiplying (17) by $U_{2}^{T}$ yields

$$
U_{2}^{T} L_{41}=U_{2}^{T} \Psi_{k}(B, D) L_{11}
$$

Since $\Psi_{k}(B, D)$ is linear with respect to $(B, D)$, we can obtain the least-squares estimate of them.

It should be noted that $\operatorname{rank}(\hat{U})=2 \mathrm{~km}$ must hold in order to apply this algorithm. But, since Assumption A3 and eq. (2) imply that $u$ has PE condition of order $2 \mathrm{~km}$, so that $u_{d}$ has also PE condition of order $2 \mathrm{~km}$.

\section{NUMERICAL EXAMPLE}

In this section, we give two numerical examples, comparing the proposed method with the joint inputoutput method (Katayama et al., 2002). In the first case, the output disturbance is a white noise, and in the second case, we assume that the output disturbance is a colored noise. A mathematical model is a slightly modified version of the one used in (Verhaegen, 1993). The transfer functions of the plant, controller and noise models are given by

$$
\begin{aligned}
& P(z)=\frac{10^{-3}\left(0.98 z^{4}+12.99 z^{3}+18.59 z^{2}+3.3 z-0.02\right)}{z^{5}-4.4 z^{4}+8.09 z^{3}-7.83 z^{2}+3.99 z-0.86} \\
& C(z)=\frac{0.63 z^{4}-2.083 z^{3}+2.8222 z^{2}-1.865 z+0.4978}{z^{4}-2.65 z^{3}+3.11 z^{2}-1.75 z+0.39} \\
& H(z)=1, \quad F(z)=0
\end{aligned}
$$

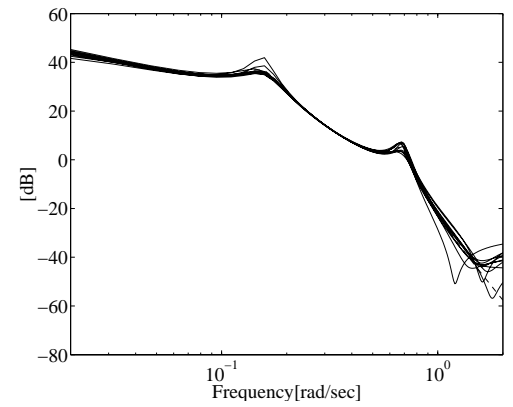

Fig. 5. Bode plots of the estimated plant by the present method (colored noise).

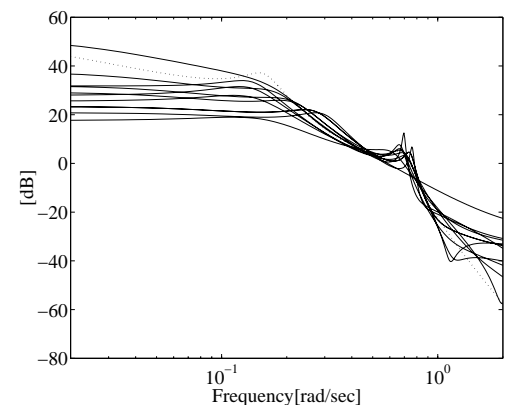

Fig. 6. Bode plots of the estimated plant by the joint input-output method (colored noise).

The inputs $r_{1}$ and $r_{2}$ are Gaussian white noises with means zero and variances $\sigma_{1}^{2}=1, \sigma_{2}^{2}=1$, and $\xi$ is a white noise with mean zero and variance $\sigma_{\xi}^{2}=0.2$.

For simulation studies, we take $N=2000, k=15$ and generated ten data sets, where in each case different Gaussian random numbers with specific variances are generated. Since $H(z)=1$, the disturbance on the output is a white noise.

Figs. 3 and 4 show Bode plots of plant estimated by the present ORT-based method and the joint inputoutput method due to (Katayama et al., 2002), respectively. We clearly see that the present method gives better results than the joint input-output method.

Now we consider the case where the output noise $v$ is a colored noise, where the noise filter is given by

$$
H(z)=\frac{z^{3}-1.56 z^{2}+1.045 z-0.3338}{z^{3}-2.35 z^{2}+2.09 z-0.6675}
$$

Other conditions are the same as before. Figs. 5 and 6 display Bode plots of plant estimated by the present method and the joint input-output method, respectively. Also, Figs. 7 and 8 display poles of the estimated plant corresponding to Figs. 5 and 6, respectively. For the case where the disturbance is a colored noise, some of the estimated poles obtained by the joint input-output method are located outside the unit circle as in Fig. 8, while those obtained by the ORTbased method are clustered near the true poles as in Fig. 7. Thus we can safely say that the present ORTbased method provides better results than the joint input-output method (Katayama et al., 2002). 


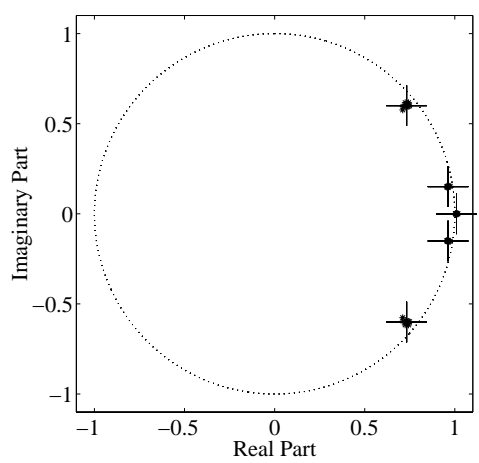

Fig. 7. Poles of the estimated plant by the present method (colored noise).

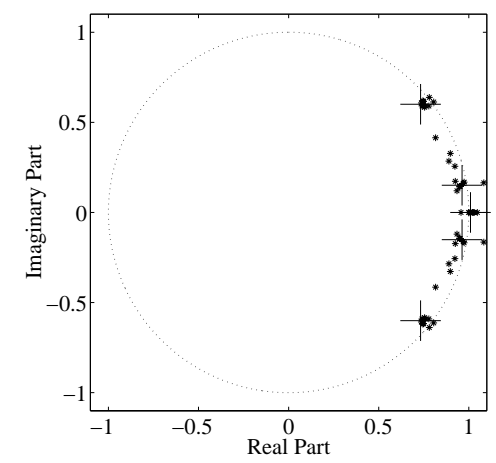

Fig. 8. Poles of the estimated plant by the joint inputoutput method (colored noise).

\section{CONCLUSIONS}

In this paper, under the assumption that the system is open-loop stable, a subspace identification algorithm is derived based on two successive LQ decompositions of a data matrix generated by exogenous inputs and plant input-outputs. The idea is somewhat related to the existing methods: the two-stage method (Van den Hof and Schrama, 1993) and the projection method (Forssell and Ljung, 1999). But, the present ORTbased method can easily be applied to multivariable systems. Also, though limited to open-loop stable closed-loop systems, the algorithm is quite simple and has high accuracy. Some simulation results show that the present method yields better numerical results compared with the method based on joint input-output approach.

\section{REFERENCES}

Chiuso, A. and G. Picci (2003). Constructing the state of random processes with feedback. In: $C D-R O M$ Preprints 13th IFAC Symposium on Systems Identification. Rotterdam. pp. 881-886.

Codrons, B., B. D. O. Anderson and M. Gevers (2002). Closed-loop identification with an unstable or nonminimum phase controller. Automatica 38(12), 2127-2137.
Forssell, U. and L. Ljung (1999). Closed-loop identification revisited. Automatica 35(7), 1215-1241.

Forssell, U. and L. Ljung (2000). A projection method for closed-loop identification. IEEE Trans. Automat. Contr. 45(11), 2101-2106.

Jansson, M. (2003). Subspace identification and ARX modeling. In: CD-ROM Preprints 13th IFAC Symposium on Systems Identification. Rotterdam. pp. 1625-1630.

Katayama, T. and G. Picci (1999). Realization of stochastic systems with exogenous inputs and subspace identification methods. Automatica 35(10), 1635-1652.

Katayama, T., H. Kawauchi and G. Picci (2002). Subspace identification of closed loop systems by stochastic realization. In: $C D-R O M$ Preprints 15th IFAC World Congress. Barcelona, Paper No. T-Mo-M02-2.

Katayama, T., H. Kawauchi and G. Picci (2005). Subspace identification of closed-loop system by the orthogonal decomposition method. Automatica 41(5), to appear.

Picci, G. and T. Katayama (1996a). A simple 'subspace' identification method with exogenous inputs. In: Proc. IFAC 13th World Congress. San Francisco, vol. I. pp. 175-180.

Picci, G. and T. Katayama (1996b). Stochastic realization with exogenous inputs and 'subspace methods' identification. Signal Processing 52(2), 145-160.

Qin, S. J. and L. Ljung (2003). Closed-loop subspace identification with innovation estimation. In: $C D$ ROM Preprints 13th IFAC Symposium on Systems Identification. Rotterdam. pp. 887-892.

Van den Hof, P. M. J. (1997). Closed-loop issues in system identification. In: Proc. 11th IFAC Symp. System Identification. Kitakyushu, Japan. pp. 1651-1664.

Van den Hof, P. M. J. and R. J. P. Schrama (1993). An indirect method for transfer function estimation from closed loop data. Automatica 29(6), 15231527.

Verhaegen, M. (1993). Application of a subspace model identification technique to identify LTI systems operating in closed loop. Automatica 29(4), 1027-1040. 